\title{
Oxazole Dyes With Potential For Photoluminescence Bioprobes: A Two-Photon Absorption Study
}

Abegão, Luis Mg; Fonseca Rodriguez, Ruben Dario; Ramos, Tárcius N; Mahuteau Betzer, Florence; Piguel, Sandrine; Joatan, José R; Mendonça, Cleber Renato; Canuto, Sylvio; Silva, Daniel Luiz; De Boni, Leonardo.

\section{Abstract}

In this work, six m-conjugated oxazole compounds dissolved in dichloromethane were characterized with linear and nonlinear optical measurements. Z-scan with femtosecond laser pulses was employed to determine the two-photon absorption (TPA) spectra. Other photophysical parameters, such as absorbance, solvatochromism, lifetime fluorescence, and fluorescence anisotropy, were evaluated with linear optical techniques. The experimental TPA cross section spectra were adjusted by the sum-over-states (SOS) model, by which important parameters such as transition dipole moments and broadening parameters were determined. To better understand the TPA spectra of the oxazole compounds, quantum-chemical calculations using the response function formalism and the density functional theory level of theory were performed. Using the results provided by the quantum-chemical calculations and the broadening parameters estimated through the application of the SOS model, the TPA spectra were simulated by the superposition (summation) of individual homogeneous Lorentzian absorption profiles.

\section{Keywords}

Density Functional Theory; Dichloromethane; Fluorescence; Laser Pulses; Optical Data Processing; Photons; Quantum Chemistry; Systems Engineering; Two Photon Processes 